\title{
Crowdsourcing Transparency Requirements through Structured Feedback and Social Adaptation
}

\author{
Mahmood Hosseini, Alimohammad Shahri, Keith Phalp, Raian Ali \\ Faculty of Science and Technology \\ Bournemouth University, UK \\ \{mhosseini, ashahri, kphalp, rali\}@bournemouth.ac.uk
}

\begin{abstract}
Transparency is one of the main requirements in business information systems with its unique characteristics, and it requires designated engineering approaches. Despite that, transparency requirements have often been studied along other mainstream requirements relating to information, such as privacy, and seldom studied as a first-class concept. In addition, the literature on transparency is mainly driven by the perspective of information providers, and a large number of stakeholders who receive or request information are usually neglected. To achieve a holistic and more efficient management of transparency requirements, we propose a conceptual framework which integrates three mechanisms of crowdsourcing, structured feedback and social adaptation. Crowdsourcing facilitates the involvement of a large, diverse group of stakeholders in transparency engineering. The use of structured feedback helps automating the process of feedback acquisition and analysis. Eventually, as transparency requirements evolve over time, social adaptation can be applied to adapt the business information system to meet the emerging transparency requirements of the stakeholders.
\end{abstract}

Keywords-Transparency Engineering, Crowdsourcing Transparency, Structured Feedback Acquisition, Social Adaptation

\section{INTRODUCTION}

In a business information system, transparency requirements are often classified as non-functional requirements (NFRs) [1]. As an NFR, stakeholders' transparency requirements should be carefully elicited and engineered because otherwise, they can adversely affect the business information system. For instance, transparency plays a vital role in building trust relationships within a business amongst its stakeholders [2], and also between the business and its stakeholders [3].

The change of the millennium and the emergence of the new generation, sometimes called the digital natives, who are advocated to be both transparent and actively seeking transparency [4] often through the use of Internet and Web 2.0 , plus the occurrence of several recent crises in the financial sector [5] and the social sector [6] which mainly arose either from the lack or abundance of transparency, have attracted the attention of several scholars to transparency. This attention, however, has not been given enough for engineering and systematically developing transparency as a requirement.

Eliciting transparency requirements can be a difficult task, as information related to transparency may be intermixed with general information requests not related to transparency [7]. Furthermore, the difficulty increases because it should be clarified what information to disclose, how this information disclosure should be regulated considering other informationrelated NFRs, such as trust, and to whom such informa- tion should be disclosed, amongst other concerns related to transparency. For example, when a teacher reveals their age to their students who have no use of that information, they are not being transparent. However, when they reveal it to Human Resources (HR), who can make decisions based on that whether to enrol them in Young Teachers' Awards Programme, they are being transparent.

Another issue regarding the engineering of transparency requirements is their evolution over time. Initially, transparency requirements can be elicited in the early stages of software development, to be embedded in the business information system-to-be. However, transparency requirements may change over time, e.g., as certain pieces of information may no longer be needed to be transparent because they are well-known by the stakeholders. Consequently, business information systems should be able to adapt to such changes.

Let us assume, in the example of the HR website, that a pop-up window opens up when they ask the employee for a certain piece of information, explaining (and therefore being transparent) why that piece of information is needed by the HR and how it can help them in their decision-making process. Some employees, however, may be uninterested to know the rationale behind such information requests (which may cause information overload for them), while others, once they have read the information, may never want to read the same explanation again with every HR request for the same piece of information (which may cause unnecessary transparency).

Furthermore, stakeholders in a business information system have different roles within the business environment. However, transparency requirements often vary not only at the role level but also at the individual level. Therefore, it is inevitable that to meet the emerging transparency requirements, more stakeholders should be engaged during the elicitation process for the discovery of their requirements, and their voices should be heard for the evolution of the business information system.

In this paper, we propose a conceptual framework for the utilisation of crowdsourcing [8], structured feedback [9] and social adaptation [10] for the elicitation and evolution of transparency requirements of stakeholders in a business information system. Crowdsourcing facilitates an approach to engage a wide set of stakeholders during these two phases of software development. Structured feedback assists the understandability and analysability of the acquired feedback in a crowdsourcing platform. Social adaptation aids the evolution of business information system by considering users' feedback as the main driving force in planning and leading adaptation. 


\section{Motivation AND BACKGROUND}

Crowdsourcing is defined as harnessing the power of a usually large, diverse crowd through an open call [11]. Crowdsourcing potentials have been investigated in general requirements engineering activities. For instance, the effects of several crowdsourcing features [12], [13] have been studied in requirements elicitation [14], and a crowd-centric requirements engineering method has been proposed which leads to requirements with higher quality and increased user satisfaction [15].

The use of crowdsourcing in enhancing transparency is investigated, and advocated to be key to examining through the information provided by official disclosures, hacks, and mashups [16]. In accordance with this, we advocate that crowdsourcing can be utilised in the engineering of transparency requirements. This is because transparency requirements are mostly at the individual level, context-dependent, and emerging requirements. Therefore, involving a larger number of stakeholders ensures more transparency requirements are elicited.

Furthermore, crowdsourcing can be seen as one solution to ensure bi-directional or dynamic transparency [17], [18], in which transparency recipients also participate in the transparency requirements specification and prioritisation by interacting with transparency providers by means of social platform activities, such as commenting, liking, etc. [19].

Structured feedback is the type of feedback provided in a form which makes it easier to aggregate, process, analyse, and evaluate. It has been investigated in crowdsourcing activities as a method of managing the large quantities of acquired feedback from the crowd. For example, the use of structured feedback in crowdsourcing activities has been explored [20], and several categories for structuring user feedback in crowdbased requirements engineering have been proposed [9].

In accordance with this, we advocate that structured feedback can improve users' transparency requirements elicitation. This can be further facilitated by the fine-grained attributes of transparency which are reflected in its facets [21]. Nonetheless, open-text feedback may still be necessary to capture elements such as users' feelings about their transparency requirements.

Social adaptation advocates that users' feedback should be regarded as the main driving force in planning software system adaptation [10], in contrast to self-adaptation, which attempts to adapt the software system by autonomously monitoring and reacting to changes in the environment. Thus, social adaptation is a means of facilitating software system evolution by responding to the collective judgement of users.

Transparency requirements are often volatile, e.g., they often change over time, and different stakeholders may require different levels of details with regards to information, or they may need various representations for the same information to help them in their understanding. Furthermore, transparency requirements of stakeholders may change when their other requirements change. For example, it is shown that as users' level of trust in an organisation increases, their transparency requirements from that organisation may decrease [22]. To this end, we advocate that the power of social adaptation, through crowdsourcing and structured feedback, can be utilised to evolve the business information system to meet the transparency requirements of its stakeholders.

\section{APPLYING CROWDSOURCING IN THE ENGINEERING OF TRANSPARENCY REQUIREMENTS}

Four reference models have been proposed for the engineering of transparency requirements [23]. In this section, we will review how crowdsourcing can facilitate the engineering of transparency requirements through these reference models.

\section{A. Crowdsourcing and Transparency Actors Wheel}

Transparency Actors Wheel identifies actors involved in transparency provision. These actors can be mapped to different pillars of crowdsourcing [12], as follows:

- Information providers can be mapped to crowdsourcers. Information providers may be individuals, organisations and governmental institutions which aim to be transparent, possibly as a regulatory requirement, by disclosing the alleged information about their stored data, their activities, and their policies to other individuals, organisations, and governmental institutions.

- Information receivers can be mapped to crowd members. They may be the individuals, organisations and governmental institutions which receive or request information and need transparency.

- Information medium can be mapped to the crowdsourcing platform. This can be the website or platform where information requests are managed and information is made accessible to those who need it.

- The act of information provision can be mapped to the crowdsourced task.

Crowdsourcing has the potential to identify the relevant stakeholders, e.g., through tools such as StakeRare [24] and StakeSource [25]. Moreover, it has the potential to identify the relevant media as well. Thus, it can be utilised in Transparency Actors Wheel for the following.

- Identification of information providers: The crowd can identify whether information is provided by the right information provider, and whether there are alternative sources to obtain information. The latter, for example, has been acknowledged in Section 2.21.1 of Freedom of Information Act as a way to make information exempt from provision by public authorities [26].

- Identification and creation of information mediums: The crowd can identify different information mediums through which information is channelled. This can lead to more information availability, which facilitates the first step towards useful transparency. They can also propagate information in new information mediums, e.g., social media and personal blogs, and help increase availability and accessibility of information to other information receivers.

- Identification of other information receivers: The crowd can identify other stakeholders who need access to the same information, thus spreading transparency to other potential stakeholders. 


\section{B. Crowdsourcing and Transparency Depth Pyramid}

Crowdsourcing can help information providers to provide and information receivers to reach the desired levels of transparency meaningfulness, which are represented in Transparency Depth Pyramid as data, process, and policy transparency. Mismatches in the provided and requested level of meaningful transparency can result in transparency failure. For example, members of the parliament in some countries publish their expenditure on designated websites for the people and media to know and investigate (data transparency). The information, however, is usually in the form of large, read-only spreadsheets (or similar) which is difficult to comprehend for the general public. Furthermore, the information may not show how the expenditure was done in term of who approved of it, where it was spent, etc. (process transparency), and also may not reveal why the expenditure was necessary and what results it achieved (policy transparency). If policy transparency is what the public wants, but is only provided with data transparency, then such a mismatch can fail transparency provision.

Crowdsourcing can help transparency meaningfulness in two ways. First, crowd members can annotate the information provided by information providers and identify information pieces as data, process or policy information. As people's annotation may vary, a simple majority role can be applied for the final visible annotation to other stakeholders. Second, crowd members can notify information providers when any part of data, process, or policy is missing, therefore enriching the data as well. The notification is facilitated by crowd members requesting more transparency on a particular piece of information, and when no other crowd member can find the requested piece of information for their peers.

\section{Crowdsourcing and Transparency Achievement Spectrum}

To achieve useful transparency, seven steps should be taken according to Transparency Achievement Spectrum [23]. Crowdsourcing can be utilised in each step to reach the final step, where transparency helps the crowd members to make informed decisions and act upon the information. Regarding each step, crowdsourcing can be utilised in the following ways:

- Information availability: The crowd can help information providers in identifying loci where the requested information is not available, e.g., by spotting places in a document where part (or all) of the information is missing. The crowd can also disseminate information through different media, such as the social media, to help it reach a wider set of stakeholders.

- Information interpretation: The crowd can interpret information to new forms which are understandable to other crowd members with different understanding capabilities (e.g., by interpreting information for people with certain disabilities), can help interpret formal interpreters (e.g., by helping news agencies in their understanding of the regional contexts where an event has happened and a piece of news on it is being produced), and can compare interpretations with each other to identify possible discrepancies and stretching of the actual information (e.g., by comparing two pieces of news and their interpretations on two different news agency websites).
- Information accessibility: The crowd can identify difficulties in accessing information, e.g., when too many clicks are needed to access the required information or when information is presented in a now obsolete electronic format. Similar to information availability, the crowd can also make the information more easily accessible by sharing it on new media.

- Information perception: The crowd can share their perception of the received information, and thus help information providers to better understand how their information, in its current representation and format, is perceived by information receivers, and whether to perceived information matches the intended perception they want information receivers to hold. Such mismatch can also drive the information providers to elicit information receivers' expectations through their feedback and represent information in a way that minimises the perception gap [27].

- Information understandability: The crowd can help raise understandability of the information by highlighting places in the information where it has ambiguities, discrepancies, etc. As different stakeholders have different understanding capabilities, a larger crowd can spot more understandability problems than a smaller crowd. For example, the crowd can restate their understanding of the provided information, preferably through the use of controlled vocabulary when possible, to compare and contrast them with other people's understanding of the same piece of information, effectively pinpointing places of ambiguity.

- Information acceptance: The crowd can contribute their reasons leading to acceptance or refusal of the provided information. Knowing the underlying reasons why information is accepted or refused is a crucial factor for information providers in transparency provision. For example, if a news agency understands the reasons behind refusing to acknowledge their news article is the lack of reputation of their source of news, they will replace their news source with a more reputable one. Since information acceptance depends on data such as people's experience and expertise and on the context where information is being provided [28], eliciting these data would also enrich information providers' understanding of why information is accepted or refused by the stakeholders.

- Information actionability: The crowd can explain how the information has helped them in their decisionmaking processes, has facilitated embarking on an action, or has changed their views and perspectives on a given topic. Stating such reasons helps other information receivers in their decision-making processes, and also helps information providers to understand the application of their transparency, facilitating future information provision as well. For example, when a bank knows that its customers used the new information about reductions in interest to transfer their money from their bank to other banks, their will take measures to reduce the effects on future transparency provision, while it helps other customers to probably take similar decisions as well. 


\section{Crowdsourcing and Information Quality in Transparency}

Information quality is divided into two groups of quality dimensions conforming to specifications and quality dimensions meeting or exceeding consumer expectations [29]. Crowdsourcing plays different roles in each of these categories:

- Product/service quality dimensions conforming to specifications: These quality dimensions are free-oferror, concise representation, completeness, consistent representation, timeliness and security. Information providers can independently guarantee these quality dimensions. However, the crowd can help find inconsistencies and imprecisions in the provided information with relation to these quality dimensions.

- $\quad$ Product/service quality dimensions meeting or exceeding consumer expectations: These quality dimensions are appropriate amount, relevancy, understandability, interpretability, objectivity, believability, accessibility, ease of manipulation, reputation, and value-added. These quality dimensions cannot be independently decided by information providers, and the involvement of the crowd is crucial to guarantee whether these qualities have been met.

\section{Applying Structured Feedback in the ENGINEERING OF TRANSPARENCY REQUIREMENTS}

User feedback and its structure have been discussed in crowd-based requirements engineering [9]. Structured feedback can be a useful tool in the elicitation and analysis of the crowd members' transparency requirements. Structured feedback can be obtained in a way that pinpoints the exact locus of transparency success or failure. The locus can be related to any of the reference models in transparency which relates to information, as follows:

- $\quad$ Structured feedback for transparency meaningfulness: The feedback may consist of the level of transparency required (data, process or policy) and the level of transparency achieved, therefore highlighting potential incompatibilities.

- Structured feedback for transparency usefulness: The feedback may consist of the last step of transparency usefulness achieved, and why the higher steps have failed to be reached.

- Structured feedback for information quality in transparency: The feedback may consist of tick boxes for all quality dimensions achieved.

Furthermore, structured feedback can be used for stakeholder identification and medium discovery. Here, the feedback can consist of stakeholders' names and their role in providing or receiving the information, and names of the media and links to them where the required information is channelled.

The use of structured feedback helps to pinpoint those transparency facets which have not been fulfilled, and every facet needs to be dealt with in a unique manner. For example, failure in providing transparency meaningfulness leads to new forms of information disclosure in which the process leading to the disclosure of information or the policy based on which information disclosure happens is also disclosed. On the other hand, failure in providing timely information does not lead to new forms of information disclosure, but to information disclosure that happens when a stakeholder needs that information.

Finally, we advocate that the feedback platform should still facilitate the elicitation of users' transparency requirements through users' comments in a free-form format. This will give users to explain their choices, and add anything they find valuable for requirements engineers in their analysis. A finegrained, more elaborated form of such structured feedback will constitute our future research.

\section{ApPlying Social AdAPtation In the ENGINEERING OF TRANSPARENCY REQUIREMENTS}

Social adaptation proposes a continuous obtaining and analysis of social feedback for adapting the software system [10]. As a result, we advocate that social adaptation can be utilised alongside crowdsourcing and structured feedback for a better management of transparency requirements.

One of the main issues to be considered in the engineering of transparency requirements is that transparency requirements are often individual, context-dependent, emerging requirements. Therefore, it is ideally the case that the engineering process should capture every individual's needs in a timely, context-aware fashion as stated in their feedback.

A possible solution, which is already in practice in production and industrial engineering, is mass customisation. Mass customisation is "the ability to provide individually-designed products and services to every customer through high process flexibility and integration" [30]. Unlike industrial products, informational products are generally easier for mass customisation once a business information system has the stakeholder's transparency requirements through feedback. Amongst other things, mass customisation of informational products mainly deals with the answers to questions such as what information to disclose and what information not to disclose, including the level of details, when to disclose the information, i.e., adhering to timeliness, who to disclose the information to, i.e., knowing the relevant stakeholders, how to disclose the information, i.e., the method of representation, and where to disclose the information, i.e., the choice of the medium.

Once these questions are answered, generating an informational report covering the necessary details and eliminating the unwanted bits, and presenting it to the relevant stakeholders will not be a difficult task, and will target the level of transparency that the stakeholder desires. That being said, the use of social adaptation and the MAPE loop should still be considered for every individual as well. Because while different stakeholders have different transparency requirements, the transparency requirements of one individual is also subject to change as the context changes or as the time passes.

\section{A. Phase One: Monitoring}

Stakeholders' transparency requirements change as time passes and as they are situated in different contexts, and their existing requirements become obsolete as they obtain the information they need, replaced by new transparency requirements (or by none, as once they obtain the information they 
need, they might not want to get the same information again). Monitoring these changes is the first step in ensuring that stakeholder's transparency requirements are appropriately met, and such monitoring requires constant feedback, preferably in a structured form, from each engaged stakeholder. The output of the monitoring phase will be a list of changes in a stakeholder's preferences.

The monitoring phase aims to utilise the power and wisdom of the crowd through structured feedback for social adaptation. It is denoted that for the wisdom of the crowd to work, four aspects of crowd diversity, independence amongst crowd members, decentralisation, and aggregation of the crowd knowledge must be considered. However, each of these aspects introduce their own benefits and challenges [31] which must be addressed by requirements engineers in any requirements monitoring framework used in this phase.

There are frameworks for monitoring requirements in enterprise systems [32], [33], which may also be used for monitoring transparency requirements. However, involvement of the users through social adaptation means that users become monitors and their feedback can act as triggers for system behaviour change. For transparency requirements, this change means changes in the volume of information, its representation, its disclosure time, etc.

\section{B. Phase Two: Analysis}

The analysis of transparency requirements should be performed individually for each stakeholder. When the obtained feedback is structured, the difficulties of such individual analysis are alleviated. Furthermore, the structured feedback facilitates automated analysis of users' feedback, based on which several alternatives for adaptation can be proposed.

We advocate that automated reasoning should be utilised during the analysis phase. In order to perform automated reasoning on transparency requirements, transparency requirements should be first expressed in a formal language, and then automated algorithmic manipulations should be applied on the formal language used for transparency requirements.

There are several frameworks for automated requirements analysis, e.g., the goal-structured analysis framework [34]. However, we advocate the need for a domain-specific modelling language for transparency and automated analysis. To this end, we have already proposed TranspLan, which is a domain-specific modelling language for transparency requirements in business information systems [23].

\section{Phase Three: Planning}

The third step in socially adapting the business information system to new transparency requirements of its users is planning the best alternative. Planning includes a careful consideration of the possible alternatives and selecting the one that satisfices [35] users' transparency needs.

We advocate utilising recommender systems to find the best alternative for meeting users' transparency requirements. The recommender system, however, should be designed considering that users' requirements and interests change over time, and it should understand users' needs at different stages [36].
Furthermore, we advocate that user profiling should be considered when possible. User profiling helps the recommender system understand what users find interesting and uninteresting [37] in transparency, and the recommender system can use this data, along with users' feedback, to plan for the best alternative which meets users' new transparency requirements.

\section{Phase Four: Execution}

Execution for change should be done in a way that does not affect the current performance of the business information system. It should be noted that unlike privacy requirements which need to be constantly met, most transparency requirements are transient, meaning that once they are met, the stakeholders may no longer be interested in the same information. This does not remove their transparency requirements, only the need for the information based on which transparency is founded.

An overview of our proposed conceptual framework for applying three concepts of crowdsourcing, structured feedback and social adaptation in the engineering of transparency requirements is illustrated in Fig. 1.

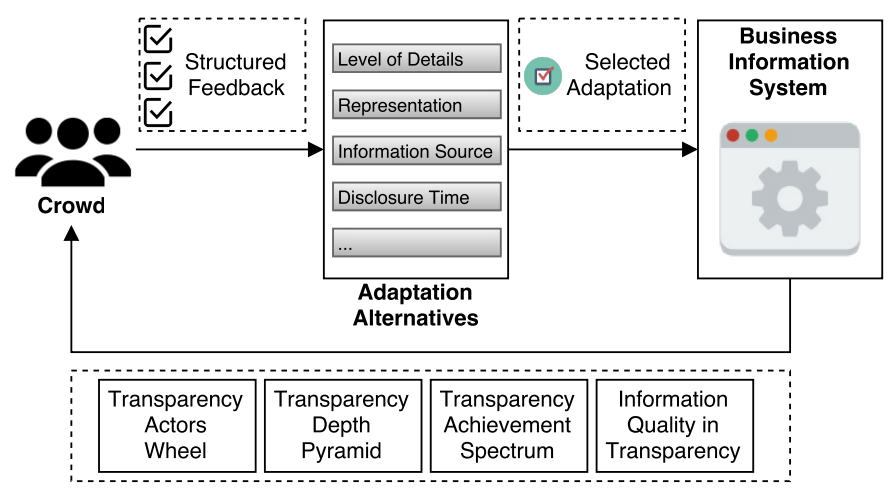

Fig. 1: An overview of the conceptual framework for crowdsourcing transparency requirements through structured feedback and social adaptation

\section{CONCLUSION}

In this paper, we proposed a conceptual framework for utilising crowdsourcing and social adaptation through structured feedback for engineering and evolving transparency requirements. We argued that this framework provides several benefits. First, crowdsourcing provides a solid ground for the engagement of the crowd in identifying and improving transparency requirements, stretching the involvement from ordinary users to potentially everyone interested in contributing to the evaluation and evolution of the business information system with regards to transparency requirements. Second, structured feedback increases the efficiency of requirements engineers dealing with transparency in pinpointing the exact loci where transparency requirements have failed or succeeded to be met, and assists their automated analysis. Third, social adaptation provides a perpetual user engagement and the selection of the best alternatives to adapt the business information system to its ever-changing transparency requirements. 
Our future work will concretise the proposed conceptual framework and provide methods supported by automated tools to cover the entire life cycle of socially-adaptive transparency requirements. This includes the structure of transparencyrelated feedback, concrete crowd-based transparency requirements elicitation methods, proved aggregation algorithms and adaptation decision making.

\section{ACKNOWLEDGMENT}

The research is supported by an FP7 Marie Curie CIG grant (the SOCIAD project).

\section{REFERENCES}

[1] J. C. S. do Prado Leite and C. Cappelli, "Software transparency," Business \& Information Systems Engineering, vol. 2, no. 3, pp. 127139, 2010.

[2] A. Bellver and D. Kaufmann, "Transparenting transparency: Initial empirics and policy applications," World Bank Policy Research Working Paper, pp. 1-72, 2005.

[3] A. Vaccaro and P. Madsen, "Corporate dynamic transparency: the new ict-driven ethics?" Ethics and Information Technology, vol. 11, no. 2, pp. 113-122, 2009.

[4] S. Casey, "How millennial are you?" PM World Journal, vol. IV(X), October 2015.

[5] G. Michener and K. Bersch, "Conceptualizing the quality of transparency," in paper apresentado na $1^{a}$ Conferência Global sobre Transparência, 2011.

[6] R. K. Ko, G. Russello, R. Nelson, S. Pang, A. Cheang, G. Dobbie, A. Sarrafzadeh, S. Chaisiri, M. R. Asghar, and G. Holmes, "Stratus: Towards returning data control to cloud users," in Algorithms and Architectures for Parallel Processing. Springer, 2015, pp. 57-70.

[7] M. Hosseini, A. Shahri, K. Phalp, and R. Ali, "Towards engineering transparency as a requirement in socio-technical systems," in IEEE 23rd International RE Conference, Aug 2015, pp. 268-273.

[8] M. Hosseini, A. Shahri, K. Phalp, J. Taylor, R. Ali, and F. Dalpiaz, "Configuring crowdsourcing for requirements elicitation," in IEEE 9th International Conference on Research Challenges in Information Science, 2015, pp. 133-138.

[9] N. Sherief, W. Abdelmoez, K. Phalp, and R. Ali, "Modelling users feedback in crowd-based requirements engineering: An empirical study," in The Practice of Enterprise Modeling. Springer, 2015, pp. 174-190.

[10] R. Ali, C. Solis, I. Omoronyia, M. Salehie, and B. Nuseibeh, "Social adaptation: when software gives users a voice," in 7th International Conference Evaluation of Novel Approaches to Software Engineering, June 2012.

[11] J. Howe, "Crowdsourcing: A definition," June 2006. [Online]. Available: http://www.crowdsourcing.com/cs/2006/06/crowdsourcing_a.html

[12] M. Hosseini, K. Phalp, J. Taylor, and R. Ali, "The four pillars of crowdsourcing: A reference model," in IEEE Eighth International Conference on Research Challenges in Information Science, May 2014, pp. $1-12$.

[13] M. Hosseini, A. Shahri, K. Phalp, J. Taylor, and R. Ali, "Crowdsourcing: A taxonomy and systematic mapping study," Computer Science Review, vol. 17, pp. 43-69, 2015.

[14] M. Hosseini, K. Phalp, J. Taylor, and R. Ali, "Towards crowdsourcing for requirements engineering," in The Empirical Track of the 20th International Working Conference on Requirements Engineering: Foundation for Software Quality, 2014.

[15] R. Snijders, F. Dalpiaz, M. Hosseini, A. Shahri, and R. Ali, "Crowdcentric requirements engineering," in IEEE/ACM 7th International Conference on Utility and Cloud Computing. IEEE, 2014, pp. 614615.

[16] J. Brito, "Hack, mash, \& peer: Crowdsourcing government transparency," The Columbia Science and Technology Law Review, vol. 9, pp. 119-157, 2008.
[17] J. Hultman and B. Axelsson, "Towards a typology of transparency for marketing management research," Industrial Marketing Management, vol. 36, no. 5, pp. 627-635, 2007.

[18] A. Vaccaro and P. Madsen, "ICT and an NGO: Difficulties in attempting to be extremely transparent," Ethics and Information Technology, vol. 11, no. 3, pp. 221-231, 2009.

[19] M. W. DiStaso and D. S. Bortree, "Multi-method analysis of transparency in social media practices: Survey, interviews and content analysis," Public Relations Review, vol. 38, no. 3, pp. 511-514, 2012.

[20] A. Xu, S.-W. Huang, and B. Bailey, "Voyant: generating structured feedback on visual designs using a crowd of non-experts," in The 17th ACM conference on Computer supported cooperative work \& social computing. ACM, 2014, pp. 1433-1444.

[21] M. Hosseini, A. Shahri, K. Phalp, and R. Ali, "Foundations for transparency requirements engineering," in The 22nd International Working Conference on Requirements Engineering: Foundation for Software Quality. Springer, 2016.

[22] A. Menéndez-Viso, "Black and white transparency: Contradictions of a moral metaphor," Ethics and Information Technology, vol. 11, no. 2, pp. 155-162, 2009.

[23] M. Hosseini, A. Shahri, K. Phalp, and R. Ali, "A modelling language for transparency requirements in business information systems," in The 28th International CAiSE Conference. Springer, 2016.

[24] S. L. Lim and A. Finkelstein, "Stakerare: using social networks and collaborative filtering for large-scale requirements elicitation," IEEE Transactions on Software Engineering, vol. 38, no. 3, pp. 707-735, 2012.

[25] S. L. Lim, D. Quercia, and A. Finkelstein, "Stakesource: harnessing the power of crowdsourcing and social networks in stakeholder analysis," in The 32nd ACM/IEEE ICSE Conference. ACM, 2010, pp. 239-242.

[26] "Freedom of information act 2000," Online [Last Accessed 21 January 2016], 2000. [Online]. Available: http://www.legislation.gov.uk/ukpga/2000/36/contents

[27] D. Tesch, R. Miller, J. J. Jiang, and G. Klein, "Perception and expectation gaps of information systems provider skills: the impact on user satisfaction," Information Systems Journal, vol. 15, no. 4, pp. 343-355, 2005.

[28] S. Watts, "Corporate social responsibility reporting platforms: enabling transparency for accountability," Information Technology and Management, vol. 16, no. 1, pp. 19-35, 2015.

[29] B. K. Kahn, D. M. Strong, and R. Y. Wang, "Information quality benchmarks: product and service performance," Communications of the $A C M$, vol. 45, no. 4, pp. 184-192, 2002.

[30] G. Da Silveira, D. Borenstein, and F. S. Fogliatto, "Mass customization: Literature review and research directions," International journal of production economics, vol. 72, no. 1, pp. 1-13, 2001.

[31] M. Hosseini, J. Moore, M. Almaliki, A. Shahri, K. Phalp, and R. Ali, "Wisdom of the crowd within enterprises: Practices and challenges," Computer Networks, vol. 90, pp. 121-132, 2015.

[32] W. N. Robinson, "A requirements monitoring framework for enterprise systems," Requirements engineering, vol. 11, no. 1, pp. 17-41, 2006.

[33] _ , "Implementing rule-based monitors within a framework for continuous requirements monitoring," in The 38th Annual Hawaii International Conference on System Sciences. IEEE, 2005, pp. 188a188a.

[34] D. Duffy, C. MacNish, J. McDermid, and P. Morris, "A framework for requirements analysis using automated reasoning," in Advanced Information Systems Engineering. Springer, 1995, pp. 68-81.

[35] L. Chung and J. C. S. do Prado Leite, "On non-functional requirements in software engineering," in Conceptual modeling: Foundations and applications. Springer, 2009, pp. 363-379.

[36] J. A. Konstan and J. Riedl, "Recommender systems: from algorithms to user experience," User Modeling and User-Adapted Interaction, vol. 22, no. 1-2, pp. 101-123, 2012.

[37] S. E. Middleton, D. C. De Roure, and N. R. Shadbolt, "Capturing knowledge of user preferences: ontologies in recommender systems," in The first international conference on Knowledge capture. ACM, 2001, pp. 100-107. 